\title{
Balkanologie
}

Balkanologie Revue d'études pluridisciplinaires

Vol. VI, $n^{\circ}$ 1-2 | 2002

Volume VI Numéro 1-2

\section{Le monastère de Sveti Prohor Pčinski}

The Sveti Prohor Pčinski monastery

\section{Bernard Lory}

\section{OpenEdition}

\section{Journals}

Édition électronique

URL : http://journals.openedition.org/balkanologie/440

DOI : $10.4000 /$ balkanologie. 440

ISSN : 1965-0582

\section{Éditeur}

Association française d'études sur les Balkans (Afebalk)

\section{Édition imprimée}

Date de publication : 1 décembre 2002

Pagination : 47-54

ISSN : 1279-7952

\section{Référence électronique}

Bernard Lory, « Le monastère de Sveti Prohor Pčinski », Balkanologie [En ligne], Vol. VI, n 1-2 | 2002,

mis en ligne le 03 février 2009, consulté le 17 décembre 2020. URL : http://journals.openedition.org/ balkanologie/440 ; DOI : https://doi.org/10.4000/balkanologie.440 


\title{
LE MONASTÈRE DE SVETI PROHOR PČINSKI
}

\author{
Bernard Lory*
}

Le processus d'affirmation nationale des Macédoniens, entre 1878 et 1945 , fut lent et contrarié. Plus que d'autres mouvements analogues dans les Balkans, il eut à surmonter des contradictions, nombreuses et intriquées, avant de parvenir à une formulation claire, recueillant l'adhésion de la masse de la population. La question, tout à fait ponctuelle, du monastère de Sveti Prohor Pčinski (Saint Prochore de la Pčinja) illustre certains aspects importants de ce processus et des renégociations identitaires qu'a imposées le $\mathrm{XX}^{\mathrm{e}}$ siècle.

Avant d'être définie comme un territoire ethnique, la Macédoine est perçue comme un territoire historique et géographique. Les érudits et voyageurs qui considèrent la Macédoine au XIX ${ }^{e}$ siècle ont en tête des références de l'Antiquité, variables, au demeurant, selon qu'ils se réfèrent au royaume de Philippe II ou au découpage provincial de l'Empire romain. Aussi, plutôt que de se raccrocher à des divisions politiques révolues, va-t-on chercher à inscrire le toponyme Macédoine dans le cadre plus rassurant et plus définitif de la géographie physique. L'orographie et l'hydrographie sont utilisées pour définir les grands sous-ensembles territoriaux de la péninsule balkanique.

L'usage s'implante d'évoquer une Macédoine géographique ou une Macédoine conventionnelle selon une logique hydrographique : elle englobe dans son territoire les bassins versants du Vardar/Axios, de la Struma/Strymon, de la Mesta/Nestos et de l'Aliakmon/Bistrica, auxquels on adjoint le bassin supérieur du Drin Noir (lac d'Ohrid, Debar). On relève cependant une entorse majeure à ce principe apparemment simple : le bassin supérieur de la Struma (région de Kjustendil) n'est pas inclus dans cette définition. Il y a là, en effet, une interférence avec une logique de nature politique : la 
Haute Struma a été attribuée par le Traité de Berlin à la Principauté de Bulgarie en 1878 et la définition géographique que l'on veut donner de la Macédoine implique que le territoire ainsi désigné soit possession ottomane (durant la période $1878-1912)^{1}$.

Cependant, n'en déplaise aux géographes en chambre, un bassin versant ne constitue pas forcément une unité naturelle. La Pčinja est un affluent du Vardar, qui, venant du nord, le rejoint en aval de Skopje. Son cours supérieur, la région de Haute Pčinja (ou Gorna Pčinja) est étroitement enchâssée entre les montagnes Kozjak au sud et Kociura au nord ; elle ne communique avec le bassin de Kumanovo qu'au travers d'une klisura (défilé épigénétique) longue et particulièrement resserrée. Aussi la sous-région extrêmement cloisonnée de Gorna Pčinja communique-t-elle plus facilement avec la région de Vranje, de l'autre côté de la ligne de partage des eaux, sur la Haute Morava ${ }^{2}$. Le village de Trgovište est considéré comme le principal centre de ce canton montagneux qui se caractérise par un habitat très dispersé.

La logique d'implantation des ermitages, d'où découle celle des monastères, est tout à l'opposé de celle des voies de communication. C'est au contraire la recherche de la solitude et de l'inaccessibilité qui la régit. Aussi le pieux ermite Prohor choisit-il pour résidence un des coins les plus sauvages de la klisura de la Pčinja. Il vécut, nous dit son hagiographie, au $\mathrm{XI}^{\mathrm{e}}$ siècle et prédit à Romain IV Diogène, alors gouverneur de Sofia et qu'une chasse effrénée avait conduit dans ces solitudes, qu'il règnerait à Constantinople ; ce règne fut d'ailleurs bref, de 1067 à 1071, et s'acheva sur le désastre de Mantzikert, lequel ouvrit toute l'Anatolie à la pénétration seldjoukide. L'ermite Prohor n'était pas seul en son genre. Il est fréquemment associé à trois autres ermites des X-XI siècles de la même région : saint Jean de Rila, saint Joachim d'Osogovo et saint Gabriel de Lesnovo, autour du culte desquels de grands monastères se développèrent. Saint Prohor et saint Jean de Rila sont d'ailleurs commémorés le même jour, le 1er novembre (19 octobre st. st.).

Le monastère Sveti Prohor Pčinski fut protégé par le roi de Serbie Milutin (1282-1311) qui fit construire une église, dont ne subsiste aujourd'hui que le mur oriental avec l'abside. Elle fut décorée de fresques de la main de Michel

\footnotetext{
${ }^{1}$ L'ouvrage de référence sur cette question de la définition territoriale qu'utilise le discours national macédonien est Makedonija kako prirodna i ekonomska celina (La Macédoine comme unité naturelle et économique), ouvrage initialement publié en bulgare en 1945, puis traduit en macédonien en 1946 (réédité ел 1978).

${ }^{2}$ Dès le XIX ${ }^{e}$ siècle, les géographes ont pu constater que la limite entre le bassin versant du Vardar (donc de la mer Egée) et ceux de l'Ibar et de la Morava (donc vers la mer Noire) était passablement floue et qu'on y observe des phénomènes de captation d'affluents dans le bassin de Kosovo Polje et autour du seuil de Preševo. La petite rivière Nerodimka présente même une bifurcation : peu avant Uroševac/Ferizaj, une partie de ses eaux s'oriente vers la Sitnica et de là vers le Danube, tandis que l'autre rejoint le Vardar par la klisura de Kačanik.
} 


\section{Sveti Prohor Pčinski}

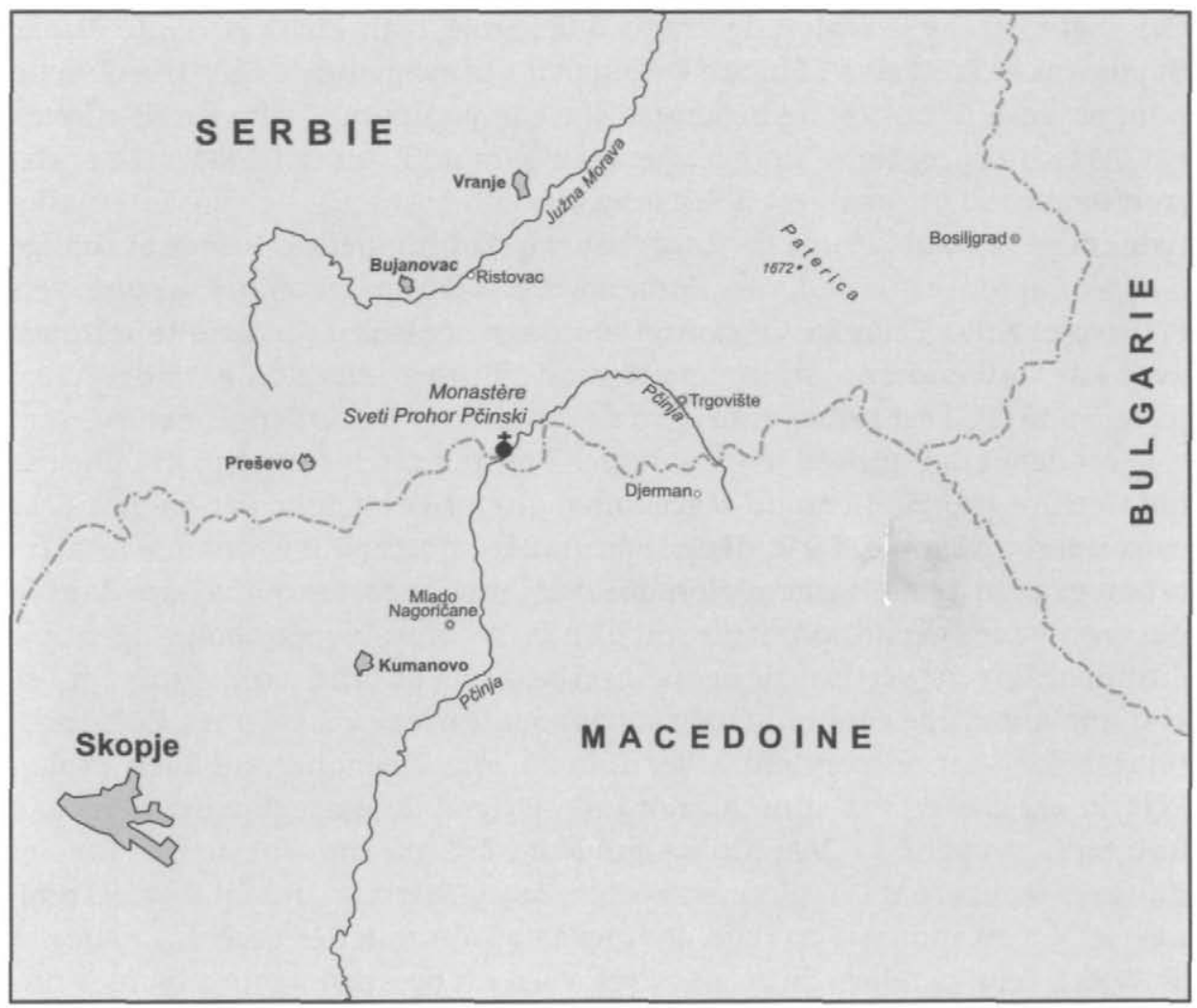

Astrapas (qui peignit à Ohrid et Staro Nagoričane). Une inscription atteste que les fresques furent refaites en 1489. Son Pomenik (registre de commémoration des donateurs) montre qu'à l'époque ottomane il était visité par des fidèles originaires d'une région grossièrement comprise entre Leskovac, Skopje et Kjustendil. On y recopia des manuscrits et on y peignit des icônes. L'église actuelle fut construite entre 1898 et 1904 .

Les difficultés politiques pour le monastère commencent à apparaître en 1878. L'armée serbe, entrée en guerre (pour la seconde fois) le 13 décembre 1877 , s'empare de Niš le 10 janvier 1878 , force la klisura de Grdelica entre le 20 et le 24 et occupe Vranje le 31 janvier. Ouand, quelques jours plus tard, le cessez-lefeu entre en vigueur, elle a atteint le nord du bassin de Kumanovo et occupe Sveti Prohor Pčinski. La ligne de démarcation officialisée le 25 février le confirme. L'arbitraire militaire va-t-il décider du sort du monastère ? Non pas, l'arbitraire diplomatique s'en charge. Le 3 mars, le traité de San Stefano attribue la région de Vranje et la Haute Pčinja à la Grande Bulgarie, laquelle n'aura 
pas d'existence réelle. Le traité de Berlin, le 13 juillet 1878 , revient sur cette décision et rattache la région de Vranje à la Serbie, mais laisse la Haute Pčinja, Bujanovac et Preševo à l'Empire Ottoman. La principauté de Bulgarie obtient, pour sa part, le canton de Bosiljgrad (qu'elle perdra au Traité de Neuilly en 1919). Les trois frontières se rejoignent sur le mont Paterica (1672 m). Le postefrontière serbo-ottoman est à Ristovac, sur la Morava. La décision internationale coupe la Haute Pčinja de son débouché traditionnel sur Vranje et l'oblige à se réorienter vers le sud, vers Kumanovo, et, dans une moindre mesure, vers Preševo et Kriva Palanka. Ce canton montagneux isolé et pauvre se retrouve avec une forte présence militaire ottomane. Il avait relevé du kaza de Vranje jusqu'en 1878 ; il est rattaché au kaza de Preševo, nouvellement constitué.

$\mathrm{Au}$ début des années 1890 la querelle entre exarchistes et patriarchistes, qui déchire les chrétiens de Macédoine, atteint ce secteur périphérique. Le consul serbe à Skopje, Karić, déploie de grands efforts pour éviter une implantation exarchiste dans une région qui revêt une importance majeure dans la perspective de l'expansionnisme serbe en Macédoine. Le métropolite de Skopje Firmiljan, fervent partisan de la cause serbe, a soin de faire reconstruire l'église et d'implanter une communauté monastique partageant ses vues. Cette politique à long terme parvient à ses fins en 1912. Le monastère Sveti Prohor Pčinski est clairement situé au nord de la ligne de partage entre Serbes et Bulgares, coupant la Macédoine du Nord-Est au Sud-Ouest. Lorsque les Bulgares occuperont la région, le 22 octobre 1915, ils massacreront les trois moines serbes du monastère. Dans le nouveau Royaume des Serbes, Croates et Slovènes, celui-ci relève du srez de Preševo. Il est desservi par des moines russes de l'émigration. Il est d'ailleurs très rarement mentionné et tout semble indiquer que c'est un lieu de dévotion strictement régional, d'accès difficile, sans aura patriotique particulière.

C'est en 1944 que les feux de l'Histoire vont converger vers ce couvent modeste et isolé. Et de fait, la plupart des livres d'histoire ne le mentionnent qu'en cette unique occasion.

Il s'agit de la Première Session de l'ASNOM (Antifašističko Sobranie na Narodnoto Osloboduvanje na Makedonija, Conseil antifasciste de Libération nationale de Macédoine), considérée comme l'acte fondateur de l'affirmation étatique de la Macédoine. Ce forum avait pour fonction de répercuter en Macédoine les mesures prises à l'échelle yougoslave par la Deuxième Session de l'AVNOJ (Conseil antifasciste de Libération nationale de la Yougoslavie) qui s'était tenue à Jajce les 29 et 30 novembre 1943. Les bases de la Yougoslavie fédérale titiste y avaient été jetées et le peuple macédonien officiellement reconnu comme peuple constitutif. Les circonstances de la guerre avaient fait que les délégués macédoniens n'avaient pas pu participer à cet acte fondateur. L'absence à Jajce ne fait que renforcer le poids symbolique de Sveti Prohor 
Pčinski. La préparation de ce forum fut longue et laborieuse. Le Comité d'Initiaitive reçut un briefing très précis au OG des Partisans, sur l'île de Vis ; rien ne fut laissé au hasard. L'ASNOM réunit finalement 122 délégués (dont certains représentaient la résistance communiste en Macédoine de l'Egée et du Pirin). Le choix de la date était hautement symbolique, le 2 août, la Saint-Elie, jour du déclenchement, en 1903, de l'Insurrection d'llinden. Quant au choix du lieu, le monastère Sveti Prohor Pčinski, à l'extrême périphérie du territoire considéré comme macédonien, il répondait surtout à des contingences stratégiques. La résistance communiste en Macédoine, ne s'étant affirmée qu'assez tardivement, ne disposait pas de vastes territoires libérés incluant de petites villes, comme les autres régions yougoslaves. Le massif du Kozjak offrait cependant un abri sûr aux délégués et le monastère des locaux suffisamment spacieux pour les réunir.

L'allocution inaugurale de l'ASNOM, faite par Panko Brašnarov, s'ouvre de façon révélatrice sur une évocation "hydrographique"de la Macédoine :

Camarades représentants populaires ! En ce moment, en ce lieu historique du St Père Prohor Pčinski, et en ce jour historique, la Saint-Elie, alors que je déclare ouvert le Premier Conseil antifasciste de Libération nationale de la Macédoine, mon âme déborde de joie et à travers mes yeux embués, je vois comment se sont mises en branle toutes les rivières, de la Pčinja au Vardar, à la Mesta et à la Bistrica [Aliakmon], éclaboussant toute la terre de Macédoine, voulant laver la honte de dix siècles d'esclavage subie par le peuple macédonien depuis la chute de l'Etat de Samuel, pour voir naître aujourd'hui un Etat macédonien nouveau, lumineux et libre3.

Derrière cette rhétorique brassant histoire, géographie et sentiment patriotique, il ne faut pas perdre de vue que, dans le contexte de 1944, l'ASNOM marque avant tout la prise du pouvoir par les communistes en Macédoine.

Les nouvelles autorités de Skopje eurent bientôt à régler un grand nombre de questions. Entre autres, celle de l'extension géographique de leur juridiction face à la Serbie. Dans un premier temps, elles eurent des prétentions sur le secteur de Bujanovac-Preševo, proposant ainsi d'admettre la frontière serbo-ottomane de 1878-1912 comme frontière inter-républicaine. A ce niveau, pourtant, la "logique hydrographique" prévalut et ce secteur de la Haute Morava fut attribué à la république de Serbie. Cette logique, en revanche, ne s'appliqua pas à la Haute Pčinja, qui, bien que relevant du bassin du Vardar, fut attribuée à la Serbie également. La raison en était que ce territoire, de par son relief, avait toujours gravité vers Vranje, beaucoup plus que vers Kumanovo. La frange méridionale de la Haute Pčinja fut néanmoins attribuée à la république de Macédoine. Les quelques villages concernés (Djerman, Nerav, Ogut et Meteževo) élevèrent d'ailleurs très tôt des protestations à ce sujet. mais les 
commissions d'enquête ne revinrent pas sur la décision initiale. Nous ne trouvons pas mention, dans ce débat, du monastère de Sveti Prohor Pčinski, qui se retrouva à la pointe méridionale du territoire serbe. Les mesures d'expropriation des domaines religieux laissèrent au monastère 24,15 ha de terres cultivables et 30 ha de forêts répartis sur les villages de Starac, Spančevac, Klenike, Klinovac et Tibužde, tous situés en république de Serbie.

Cette délimitation inter-républicaine opérée à la fin de 1945 suscita de faibles réactions ; il est vrai qu'à cette époque le pouvoir fédéral était prépondérant et que le yougoslavisme primait sur les prétentions nationales. Svetozar Vukmanović-Tempo le rappelait avec force à la fin de décembre 1945 :

Les partisans macédoniens et les partisans serbes se disputent pour un fichu village du Kozjak, à qui est ce village aux Macédoniens ou aux Serbes (...) On a l'impression que l'ennemi à réussi à pénétrer dans nos rangs et à semer la discorde. Mais notre but est de dire très fermement à tous que la Macédoine est macédonienne et yougoslave, que la Serbie est serbe et yougoslave. Quant à ce village du Kozjak, c'est un village de la Yougoslavie titiste, qu'il soit en Macédoine ou bien en Serbie4.

D'autre part il faut rappeler que les communistes macédoniens sont dans une situation délicate par rapport à leurs collègues yougoslaves : leur résistance a été tardive et peut se targuer de moins de hauts faits héroïques, leur république est pauvre, donc dépendante, et surtout ils nourrissent, dans ces années d'après-guerre le rêve de reconstituer la Grande Macédoine, rêve à côté duquel tous les autres intérêts sont secondaires.

Les décennies suivantes voient s'opérer un changement progressif. Le rêve de la Grande Macédoine s'évanouit en 1948. Les pays voisins (Bulgarie, Grèce, Albanie) servent de repoussoirs idéologiques au jeune nationalisme macédonien. La seule orientation géopolitique permettant le développement de la petite république fédérée est l'ouverture vers le nord, donc une collaboration étroite avec la Serbie. L'effort national se concentre sur l'éducation et la culture, forgeant peu à peu une identité macédonienne vivace et convaincante.

Pour la parachever, selon la logique des millet héritée de l'Empire ottoman, qui tend à faire coïncider les entités nationales avec les entités confessionnelles, les Macédoniens souhaitent se séparer du Patriarcat de Belgrade. Le 18 juillet 1967, l'autocéphalie de l'Archevêché d'Ohrid, abolie exactement 200 ans plus tôt, est rétablie. C'est un acte unilatéral, qui marque la rupture avec l'Eglise orthodoxe serbe et qui sera réprouvé par l'ensemble des églises orthodoxes. La hiérarchie macédonienne dissidente jouit de l'appui des autorités de Skopje, toutes communistes qu'elles soient. L'église serbe, sans relais dans la 
sphère politique, ne peut que camper sur ses positions. Or c'est elle qui possède légalement le monastère Sveti Prohor Pčinski.

Un élément trivial intervient vers la même époque : une route est construite, qui relie Mlado Nagoričane à Bujanovac, en passant par Sveti Prohor. La partie inférieure de la klisura de la Pčinja se trouve accessible et de forts lacets permettent de gagner le bassin versant de la Morava. Du coup, l'accès au monastère devient facile à partir du territoire macédonien (36 km depuis Kumanovo). L'afflux de fidèles, lors de la fête patronale, devient considérable, les recettes du monastère également (20 à 30000 dinars pour cette seule journée, vers le milieu des années 1970). Mais ne nous trompons pas! Nous sommes en régime communiste et la fréquentation du monastère est largement adultérée d'aspects politiques. Un musée de l'ASNOM a été installé dans les bâtiments conventuels. La "dévotion communiste" sert-elle d'alibi au culte du saint, ou bien l'inverse ? Et vers la fin des années 1980, alors que monte un nationalisme serbe qui investit le patrimoine monastique d'un rôle identitaire fort, les moines serbes de Sveti Prohor Pčinski supportent mal le rôle de gardiens de la mémoire communiste macédonienne que l'on prétend leur faire jouer. Le nationalisme macédonien, de son côté, devient plus revendicatif à mesure que l'idéologie yougoslaviste faiblit. En avril-mai 1990, le principal quotidien Nova Makedonija publie un feuilleton en 22 épisodes consacré à la question de Sveti Prohor Pčinski, qui sera repris sous forme de brochure. Dans la Fédération yougoslave ébranlée, nombreux sont ceux qui rêvent de redécoupages territoriaux. Notons que les revendications macédoniennes ne portent que sur le monastère, et non sur la région de Haute Pčinja entière (la définition "hydrographique" de la Macédoine a perdu tout son sens avec l'abandon du rêve grand-macédonien).

La proclamation de l'indépendance de la République de Macédoine, le 17 septembre 1991 modifie profondément le regard porté officiellement sur la Première Session de l'ASNOM. Son aspect communiste s'efface progressivement pour faire place à l'aspect državnotvoren (créateur de légitimité étatique). Peuple sans passé étatique, les Macédoniens, s'ils ne se raccrochent pas au royaume de Samuel (IX- $\mathrm{X}^{\mathrm{e}}$ siècle) ou à l'éphémère république de Kruševo (1903), n'ont pas d'autre référence historique que l'ASNOM. Mais il faut à tout prix en gommer le contexte yougoslave pour ne retenir que la volonté d'affirmation nationale : le passage du peuple à la nation.

La fonction symbolique de Sveti Prohor Pčinski comme berceau historique de l'Etat macédonien n'en devient que plus forte, de même que la frustration de voir le monastère se dresser à quelques centaines de mètres du poste-frontière de Jažince. La rectification territoriale serait minime en termes de superficie. Mais l'interlocuteur n'est pour l'instant pas le gouvernement de Belgrade, c'est l'Eglise orthodoxe serbe, qui ne saurait en 
54 / Balkanologie VI (1-2), décembre 2002, p. 47-54

aucun cas traiter avec des schismatiques. La solution du micro-conflit de Sveti Prohor Pčinski passe donc par la reconnaissance canonique de l'autocéphalie de l'Eglise de Macédoine. Or rien n'est plus lent que la diplomatie ecclésiastique, agissant sub specie aeternitatis.

\section{SOURCES UTILISÉES :}

Džikov (Stavre), Sveti Prohor Pčinski, Skopje, 200o. Principale source de cet article.

Trifunovski (Jovan), Gornja Pčinja, Belgrade, 1964, 248 p. Etude de géographie humaine, pourvue d'une carte détaillée fort précieuse.

Peric (Miodrag), Istorisko-pravnite aspekti na odnosite pomegju Srpskata pravoslavna crkva $i$ Makedonskata pravoslavna crkva (Aspects historico-religieux dans les rapports entre l'Eglise orthodoxe serbe et l'Eglise orthodoxe macédonienne), Skopje, 1998, 341 p. (Le dernier chapitre).

Tanasijević (Axhimandrit Pajsije), Prepodobni otac naš Prohor Pčinjski životočivi čudotvorac (Notre révérend père Prohor Pčinjski, thaumaturge et source de vie), Manastir Prepodobnog Prohora Pčinjskog, 1999, $107 \mathrm{p}$. Fournit un point de vue ecclésiastique serbe. 